\title{
Research on the Effect of the Base Cavity with Different Length Upon the Projectile
}

\author{
Lu Haibo* \\ Nanjing Artillery Academy \\ Nanjing, China \\ e-mail: lhbboo@sohu.com \\ * Corresponding Author \\ Tian Shiying \\ Nanjing Artillery Academy \\ Nanjing, China \\ e-mail:njpysy@yahoo.com
}

\author{
Shi Xianrui \\ Nanjing Artillery Academy \\ Nanjing, China \\ e-mail: shixianrui65356@126.com \\ Gan Quan \\ Nanjing Artillery Academy \\ Nanjing, China \\ e-mail: qgan446@aliyun.com
}

\begin{abstract}
Base cavity configuration is a useful choice to reduce the base drag of projectiles. This paper focuses on the influence of the cavity length on the projectile with base cavity. Base cavity with different length, including terminal one, are investigated numerically. The flow field parameters (Mach number, stream line, temperature and pressure), aerodynamic drag coefficient of the projectile with different length base cavity under a supersonic flow condition is obtained. Numerical results show that the recirculation region which is formed inside the base cavity plays a pivotal role for the reduction of the drag. With the increasing of the cavity length, the aerodynamic drag of the projectile reducing monotonously, even the length increases into a terminal one. If others influencing factors (such as charge mass, structural strength, etc.) are considered, a deeper base cavity is benefit to reduce the aerodynamic drag of the projectile and increase the range of fire.
\end{abstract}

Keywords-projectile with base cavity; terminal length; flow field; aerodynamic drag; numerical simulation

\section{INTRODUCTION}

Base cavity has been testified to be an effective configuration to reduce the aerodynamic drag of the projectile which flight under a supersonic velocity $[1,2]$. Several investigations have been done in this field [3-6].

In this paper, with some others influencing factors are ignored, such as charge mass, structural strength, etc, only the influence of the length of the base cavity on the flow field and aerodynamic drag of the projectile is paid attention to.. Four kinds of base cavity shape with different cavity length $(10,16,20,30 \mathrm{~cm})$ are supposed. Projectile with the different length base cavity, even terminal ones, are studied numerically, and the influence of the cavity length is discussed.

\section{NUMERICAL METHOD}

\section{A. Governing equation and grid}

An axisymmetric Navier-Stokes equation [7] with $k-\varepsilon$ turbulence model [8] is used as governing equations. The axisymmetric Navier-Stokes equation is given by

$$
\begin{aligned}
& \frac{\partial U}{\partial T}+\frac{\partial F(U)}{\partial x}+\frac{\partial G(U)}{\partial r}+\frac{1}{r} \cdot \frac{\partial H(U)}{\partial r} \\
& =\frac{1}{\operatorname{Re}} \cdot\left(\frac{\partial F_{v}(U)}{\partial x}+\frac{\partial G_{v}(U)}{\partial r}+\frac{1}{r} \cdot \frac{\partial H_{v}(U)}{\partial r}\right)
\end{aligned}
$$

where $U$ is the conservation variable, $F, G$ and $H$ are the inviscid terms, $F_{v}, G_{v}$ and $H_{v}$ are the viscous terms.

The Van Leer splitting method [9] is used to approximate the convective terms and the central difference method is used for the viscous terms. The time integration is solved by the LU-SSOR [10] scheme.

The two-dimensional axisymmetric boundary-fitted grids for the projectile with base cavity (cavity length $L=16 \mathrm{~cm}$ ) are shown as Fig. 1 .

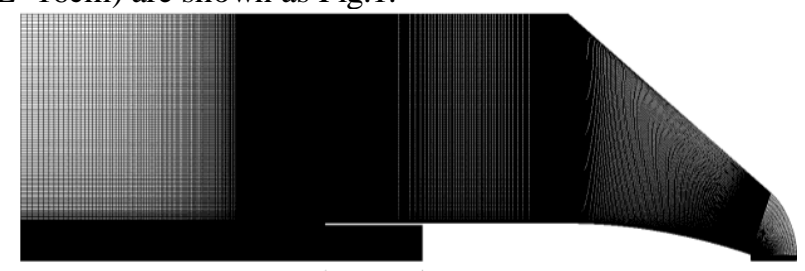

Figure 1. Grid of the simulation model (cavity length $16 \mathrm{~cm}$ )

\section{B. Boundary condition and numerical assumption}

As shown in TABLE 1, the flow field of the projectile is a supersonic one and the wall boundary condition is assumed to a no-slip and adiabatic one. 
TABLE I. BOUNDARY CONDITIONS

\begin{tabular}{ccc}
\hline Free stream parameter & Unit & Value \\
\hline Mach Number $(M a)$ & ---- & 1.97 \\
Pressure $\left(p_{\infty}\right)$ & $\mathrm{Pa}$ & 101325 \\
Temperature $\left(T_{\infty}\right)$ & $\mathrm{K}$ & 300 \\
\hline
\end{tabular}

\section{RESULTS AND DISCUSSION}

In order to discuss the influence of the length of the base cavity on the aerodynamic drag, projectile with different length $(10,16,20,30 \mathrm{~cm})$ base cavity is simulated. Of cause, a projectile with base cavity length $30 \mathrm{~cm}$, it is too large to insure the charge mass of the projectile. But in this investigation, only the influence of the cavity length is focused on. So, the paranormal projectile shape is supposed.

\section{A. Flow field}

\section{1) Mach number}

In Fig.2-Fig. 5, the distributions of Ma (with streamlines) are given. A classical bow shock is formed in front of the projectile. At the back of the projectiles, there is a recirculation region formed in the base cavity. With the cavity length increasing, the recirculation region become larger and enters into the projectile deeply.

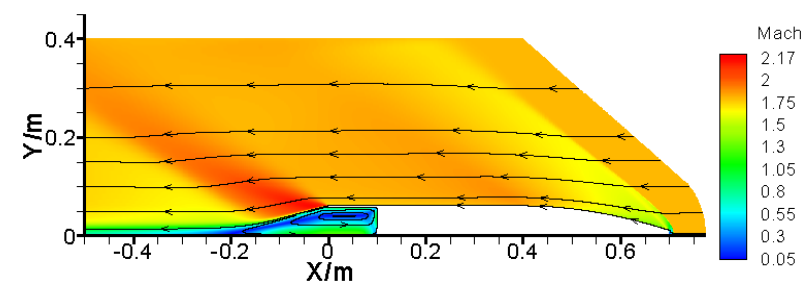

Figure 2. $M a$ (streamlines) distribution of projectile with base cavity length $10 \mathrm{~cm}$

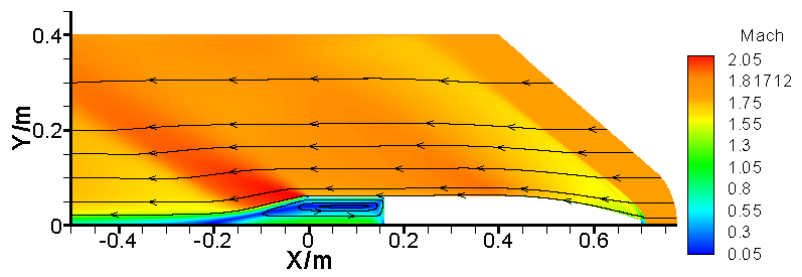

Figure 3. $M a$ (streamlines) distribution of projectile with base cavity length $16 \mathrm{~cm}$

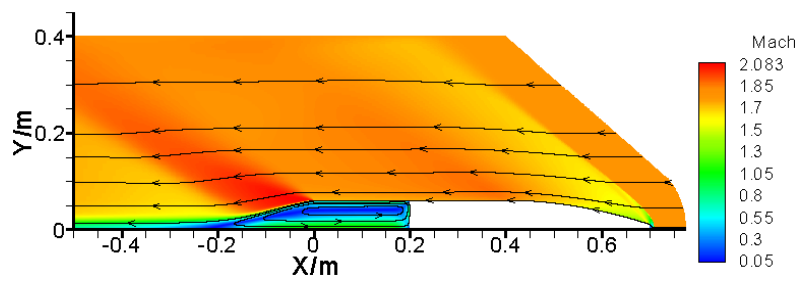

Figure 4. $M a$ (streamlines) distribution of projectile with base cavity length $20 \mathrm{~cm}$

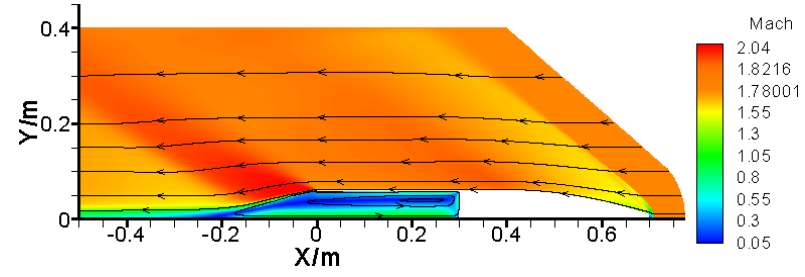

Figure 5. $M a$ (streamlines) distribution of projectile with base cavity length $30 \mathrm{~cm}$

\section{2) Temperiture}

The distribution of the flow field temperature for the projectile with different cavity length is shown in Fig. 6Fig. 9 There is a high temperature area behind the projectile and the maximum of the temperature is appeared in this region.

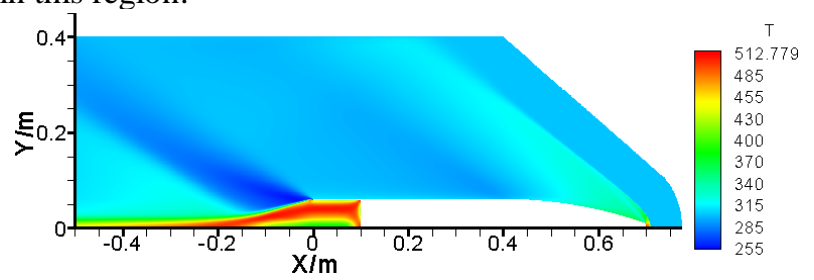

Figure 6. Temperature distribution of projectile with base cavity length $10 \mathrm{~cm}$

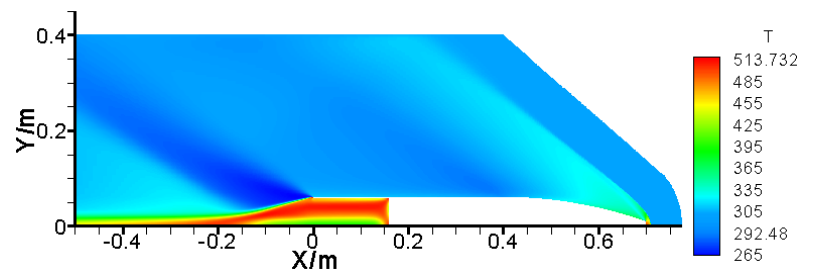

Figure 7. Temperature distribution of projectile with base cavity length $16 \mathrm{~cm}$

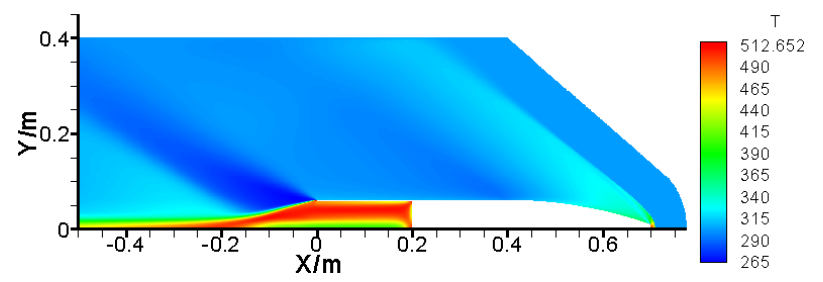

Figure 8. Temperature distribution of projectile with base cavity length $20 \mathrm{~cm}$

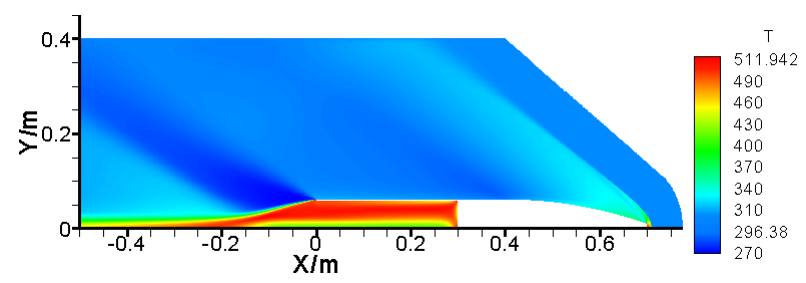

Figure 9. Temperature distribution of projectile with base cavity length $30 \mathrm{~cm}$

\section{3) Pressure}

The distributions of the pressure of the projectile with different cavity length are shown in Fig. 10-Fig. 13. As shown in these figures, inside the cavity, the distribution of the pressure of each projectile is similar. Because of the recirculation region inside the base cavity, the back 
pressure of the projectile is increased, then, the aerodynamic drag of the projectile is reduced.

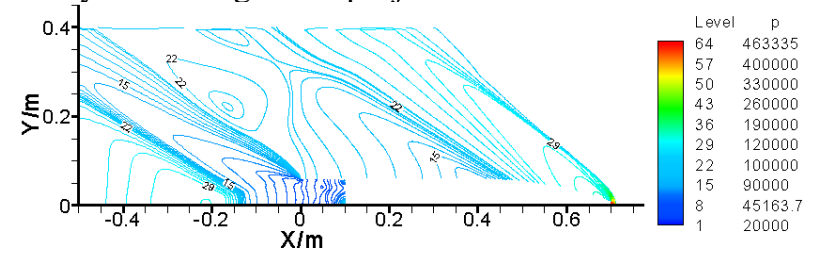

Figure 10. Pressure distribution of projectile with base cavity length $10 \mathrm{~cm}$

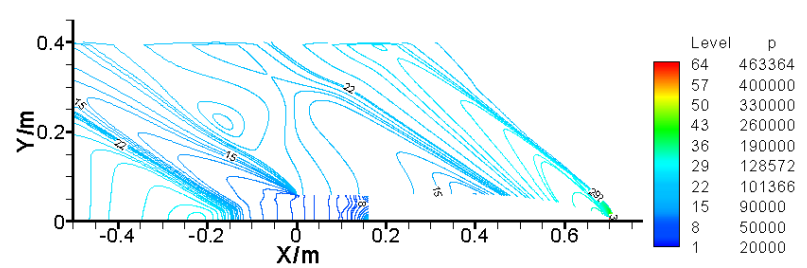

Figure 11. Pressure distribution of projectile with base cavity length $16 \mathrm{~cm}$

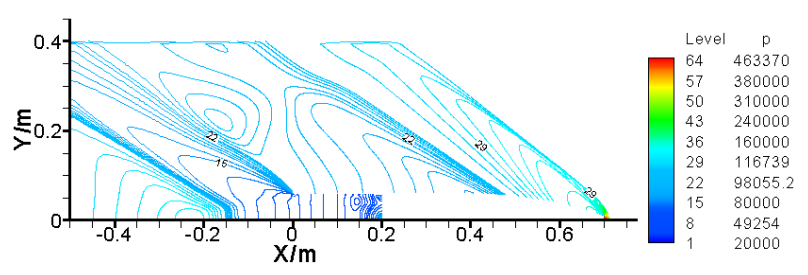

Figure 12. Pressure distribution of projectile with base cavity length $20 \mathrm{~cm}$

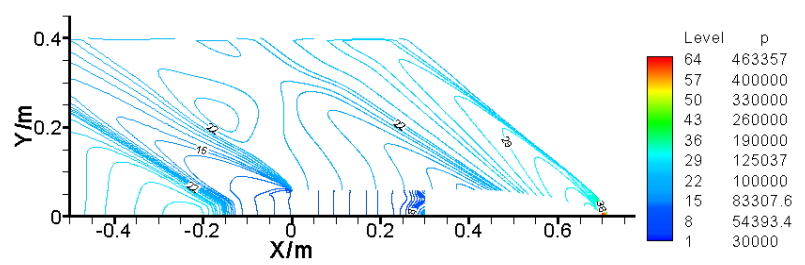

Figure 13. Pressure distribution of projectile with base cavity length $30 \mathrm{~cm}$

The distribution of pressure with stream lines inside the base cavity (length $30 \mathrm{~cm}$ ) is given un Fig. 14, which is amplified from Fig. 13. There are two regional high pressure areas along the base wall of the cavity. As stream lines shown in Fig. 5, at these regional high pressure areas, the stream lines are turning. When the back flow is turning, the velocity of the flow reduces, then the recovery of the pressure increase. In fact, there are two regional high temperature areas at the same places too (Fig. 6-Fig. 9).

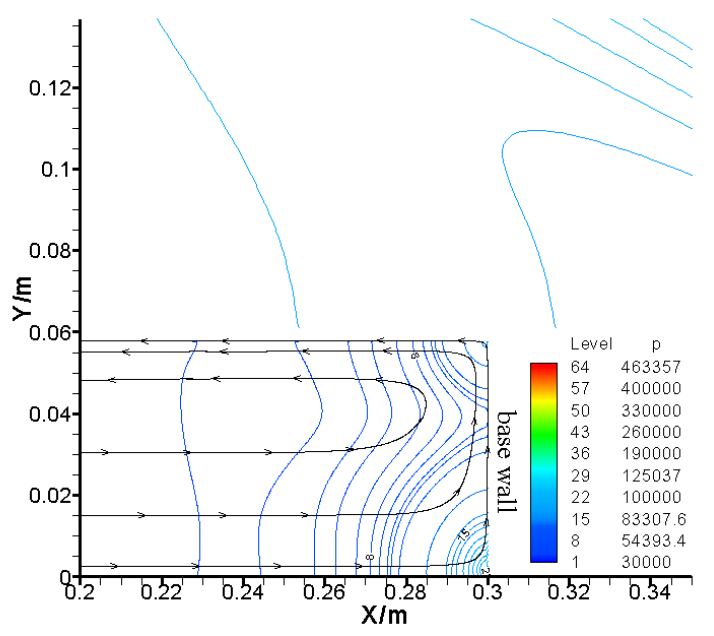

Figure 14. Pressure and stream line distributions inside the cavity $(L=30 \mathrm{~cm})$

\section{B. Aerodynamic drag}

The aerodynamic drag coefficient $\left(C_{d}\right)$ is given by

$$
C_{d}=F_{d} /\left(\frac{1}{2} \rho_{\infty} u_{\infty}^{2} \cdot S_{r e f}\right)
$$

where $F_{d}$ is the aerodynamic resistance, $\rho_{\infty}$ is the density of the free stream, $u_{\infty}$ is the velocity of the free stream, $S_{r e f}$ is the reference area which is the cross section of projectile.

The aerodynamic drag coefficients $\left(C_{d}\right)$ of the projectile with different length base cavity are shown in TABLE 2. With the increasing of the cavity length, even the length change into a paranormal level, the drag of the projectile is still reducing. It is obvious that the recirculation region plays a key role for the reduction of the drag. With the length of the base cavity increasing, the recirculation region become larger, near the base wall of the cavity, the velocity of the circumfluence is decreasing and the pressure is increasing, then, the base drag is reduced.

TABLE II. DRAG COEFFICIENT $\left(C_{d}\right)$

\begin{tabular}{ccccc}
\hline Cavity length (cm) & 10 & 16 & 20 & 30 \\
\hline $\mathrm{Cd}$ & 0.3267 & 0.3192 & 0.3161 & 0.3107 \\
\hline
\end{tabular}

With the same front shape and the same flow condition, the aerodynamic force of the projectiles is decided by their back pressure. The pressure distribution of each projectile along the base wall is given in Fig. 15. As shown in Fig. 15, it is interesting that the pressure of the regional high pressure which is located at the center of the base wall, projectile with shorter cavity is larger than the deeper one, but the pressure of the regional high pressure which is located at the corner of the base wall, their trend is just opposite. 


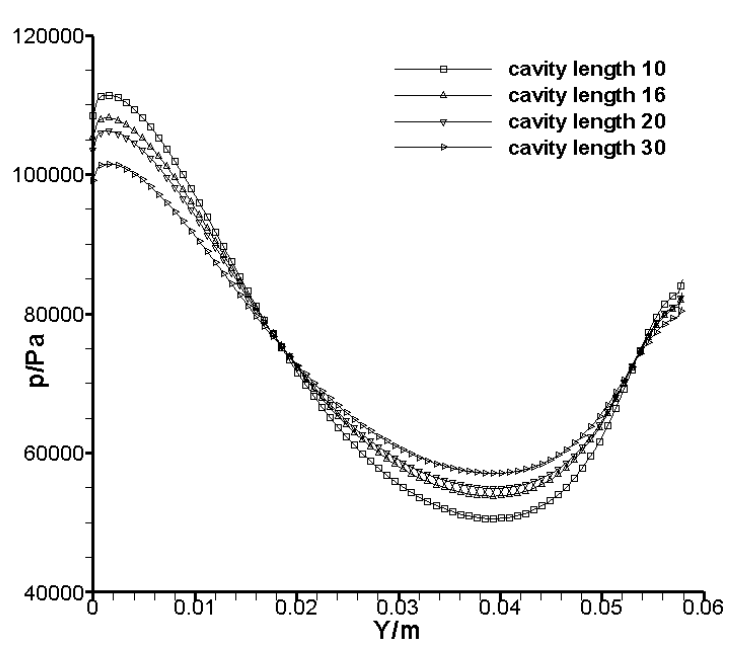

Figure 15. Pressure distribution along the base wall

\section{CONCLUSION}

This paper focuses on the influence of the cavity length on the projectile with base cavity. Projectile with different length base cavity (including paranormal one) are investigated numerically. The results show that with the increasing of the cavity length, the aerodynamic drag of the projectile reducing monotonously, even the length increases into a terminal one. If others influencing factors (such as charge mass, structural strength, etc.) are considered, a longer base cavity is benefit to reduce the aerodynamic drag of the projectile and increase the range of fire.

The flow condition of the reflux near the cavity base wall determines the back pressure of the projectile. The size of the base cavity has a great effect on the recirculation region which is inside it. The recirculation region plays a pivotal role for the reduction of the drag.

\section{ACKNOWLEDGMENT}

This work was co-supported by the National Science Foundation of Jiangsu Province, China (No. BK20130084)

\section{REFERENCES}

[1] Wei Huizhi, Zhu Hesong. "The design theory of projectile," Beijing, National Defense Industry Press, 1985 (in Chinese)

[2] Howell Jeff, Sims-Williams Daid, Sprot Adam, Hamlin Fred, Dominy Robert. "Bluff body drag reduction with ventilated base cavities," SAE Technical Papers, Vol. 5, May. 2012, pp. 152-160.

[3] Wang zhongyuan. "The effect about projectile of base cavity with side holes to flight drag under supersonic flow," ACTA Aerodynamic Sinica, vol. 15, Apr. 1997, pp. 502-506. (in Chinese)

[4] A. Ibrahim, A. Filippone. "Supersonic aerodynamics of a projectile with slot cavities," Aeronautical Journal, Vol. 114, Jan. 2010, pp. $15-24$.

[5] Wang zhongyuan. "Analysis of Base Drag Reduced by Side Holes in the Projectile with Base Cavity," Journal of Nanjing University of Science and Technology, vol. 21, Jan. 1997, pp. 17-20. (in Chinese)

[6] Cao Jiayi, Lu Chuanjing, Chen Ying, Chen Xin, Li jie. "Research on the base cavity of a sub-launched projectile," Journal of Hudrodynamics, Vol. 24, Apr. 2012, pp. 244-249.

[7] Wu Xiaoping, Wang Jianwen, Wu Xiaosong, Di Guangyou. "The numerical computation for external flow field of artillery projectile with side jet flow," Journal of Propulsion Technology, Vol. 19, Jun. 1998, pp. 57-60. (in Chinese)

[8] Tao Wenquan. "Numerical Heat Transfer," 2nd edn. Xi' an, Xi'an Jiaotong University Press. 2001 (in Chinese)

[9] Wang Xuede, Tan Junjie, Lin Xiaohong, Tang Zhenhua. "Research on parallel numerical simulation of N-S equations based on Van Leer+AUSM scheme," Jounal of Astronautics, Vol. 31, Apr. 2010, pp. 986-992. (in Chinese)

[10] Wang CY, Wang ZH, Yang XH. "Computational fluid dynamics and parallel algorithm," Changsha, National University of Defense Technology Press. $2000 \quad$ (in Chinese) 